\title{
Influence of absorption saturation on the shape of CdSe absorption edge
}

\author{
M.R. Kulish ${ }^{1}$, M.I. Malysh ${ }^{2}$, G.L. Isaienko ${ }^{3}$, V.M. Litvinova ${ }^{4}$ \\ ${ }^{1} V$. Lashkaryov Institute of Semiconductor Physics, NAS of Ukraine \\ 41, prospect Nauky,03028Kyiv, Ukraine; e-mail:n_kulish@yahoo.com \\ ${ }^{2}$ National Transport University, 1, Suvorov str., $010 \overline{10}$ Kyiv, Ukraine \\ ${ }^{3}$ National Transport University, 42, Kikvidze str., 01103 Kyiv, Ukraine, \\ ${ }^{4}$ Staten Island Technical High School, 485 Clawson Street, 10306, New York, U.S.A
}

\begin{abstract}
Influence of light polarization on absorption saturation for wurtzite modification CdSe has been investigated. It has been ascertained that the size of blurring the fundamental absorption edge for wurtzite modification CdSe is determined by the non-equilibrium electron filling both the conduction band extremum, when the energy of photons $h v$ is higher than the bandgap energy $E_{g}$, and the density-ofstate tail caused by the presence of static disorder, when the $h v<E_{g}$. The density-of-state tail resulting from the presence of dynamic disorder is not filled with electrons because of continuous changes of the dynamic potential relief value in time. In the high-energy spectral region, change in the absorption coefficient is limited by the stimulated emission processes.
\end{abstract}

Keywords: cadmium selenide, absorption edge, absorption saturation, bandwidth.

Manuscript received 18.02.14; revised version received 05.06.14; accepted for publication 29.10.14; published online 10.11.14.

\section{Introduction}

The CdSe absorption spectrum at low levels pumping (field of linear optics) of monocrystalline samples was studied in detail (see, for example [1,2]). It is known that in this case the fundamental absorption edge blurring is caused by the presence of phonons and point defects (see, for example [3-5]). Peculiarities of the dependence of absorption coefficient $K$ on excitation intensity $I_{0}$ is also researched for a certain set of wavelengths $\lambda$ (see, for example $[6,7]$ ). For this reason, the complete information about the shape of the fundamental absorption edge in the absorption saturation state was absent. Results of this work compensated the vacancy in this research field. Reported are the features of the absorption edge obtained in the framework of linear optics (when light absorption is not dependent on light intensity) and in the absorption saturation state (when pump intensities are sufficient to attain a state of absorption independence of light intensity).

\section{Samples and methods of research}

Planar lamellar $n$-type CdSe crystals were investigated. The thickness of the crystals under study was within 1 up to $400 \mu \mathrm{m}$. Their resistivity was about $1 \mathrm{Ohm} \cdot \mathrm{cm}$. The crystal optical axis was located in the plane of the sample. In the linear optics region the CdSe absorption spectrum (Fig. 1, curves 1, 3) was measured using the SDL-1 spectrometer and 100-W incandescent lamp. The absorption coefficient was determined using the Bouguer-Lambert formula: 

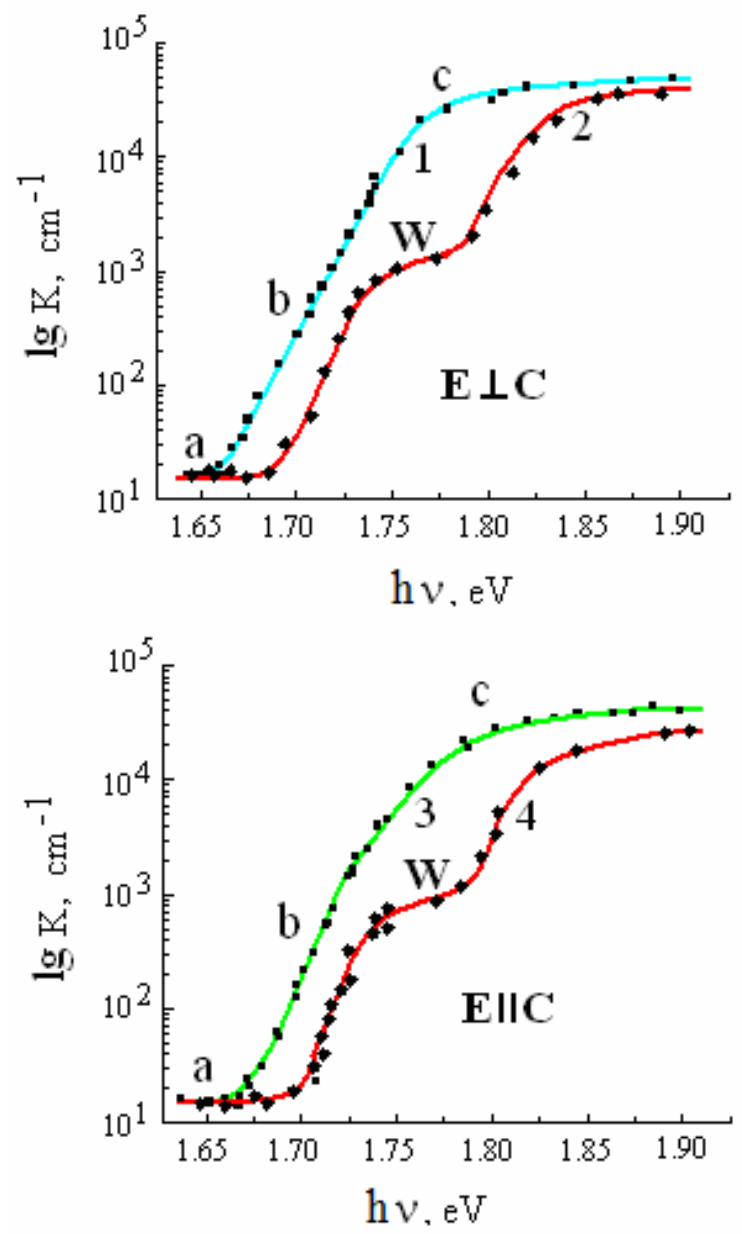

Fig. 1. Spectra of linear $(1,3)$ and nonlinear $(2,4)$ absorption of CdSe for polarizations $E \perp C(1,2)$ and $E \| C(3,4)$. $a-$ region of low intensity, $b$ - Urbach's region, $c$ - region of interband transitions, $W$ - transition region.

$I=(1-R)^{2} I_{0} \exp (-K d)$,

where $R$ is the reflection coefficient (measured for each wavelength of pumping), $K$ is the absorption coefficient, $I_{0}$ and $I$ are the light intensities at the entrance and exit of the sample, respectively, and $d$ is the sample thickness.

Influence of the pumping intensity on the transmission $T=I / I_{0}$ of CdSe samples was studied using the single-beam technique. A dye laser was used for pumping. The laser pulse duration was $20 \mathrm{~ns}$, and the spectral width of generation lines was $0.04 \mathrm{~nm}$. Light intensities at the entrance $I_{0}$ and exit $I$ of the sample were measured to determine the absorption coefficient. Change in the pumping intensity $I_{0}$ was made by reversing the calibrated neutral-gray filters before and after the sample. To determine the $I_{0}$ and $I$ values, the pulse energy, pulse duration and cross-section of the light flux incident on the sample were measured. The pulse energy was determined by a thermal calorimeter of ICT-1M type. The pulse shape was controlled by a C8-
12 oscilloscope. The cross-section of the light flux incident on the sample was determined by the conventional boundaries method. The Glan prism determined the state of light polarization relative to the optical axis. To determine the nature of influence of pumping on the absorption coefficient, we measured the dependence of the transmission $T$ on the intensity $I_{0}$ for a set of wavelengths (Fig. 2). For the wavelengths of the linear absorption spectrum in the Urbach region and interband transitions, the nonlinear $T$-dependence on $I_{0}$ was observed (Figs $2 \mathrm{~b}$ and $2 \mathrm{c}$ ). The experimental dependences were described by the equation:

$\left(I_{0} / I^{*}\right)(T-1)+\ln T=-K_{n} d$,

where $I^{*}$ is the parameter of nonlinearity. In the region of long-wave absorption tail, the experimental dependences of $T$ on $I_{0}$ were described with a straight line parallel to the $x$-axis (Fig. 2a).

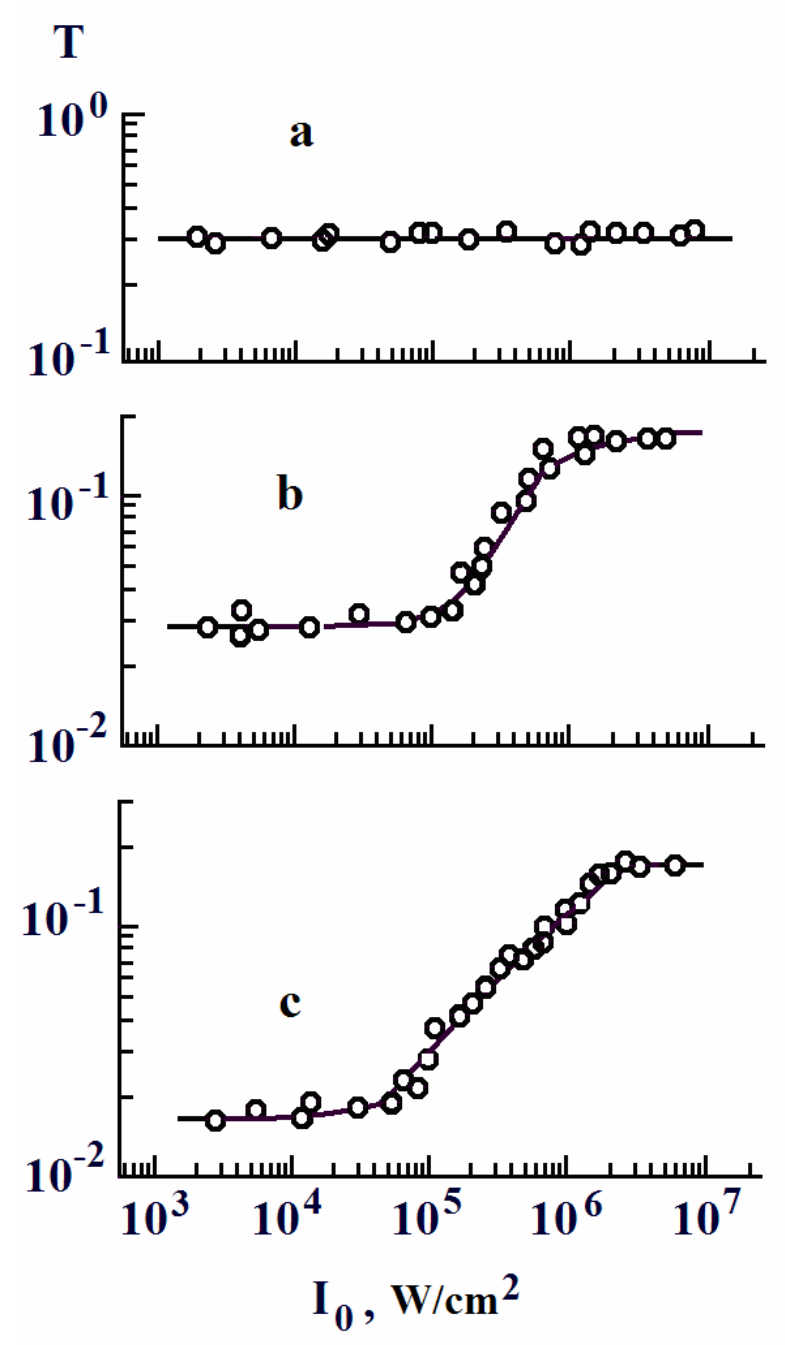

Fig. 2. Dependence $T\left(I_{0}\right)$ in the region of the long wave absorption tail $(a)$, in the Urbach region $(b)$ and that of interband transitions (c) for polarization $E \perp C$. Points is experiment, solid curves is calculated using the formula (2).

\section{(C) 2014, V. Lashkaryov Institute of Semiconductor Physics, National Academy of Sciences of Ukraine}




\section{Edge absorption spectrum in the field of linear optics}

When any technology is used for synthesis of twocomponent semiconductors, a possibility of doping these semiconductors the anion or cation clusters always exists. These clusters can absorb light photons, but their transition to the ground state is nonradiative. In addition, they can be centers of light scattering. These clusters are the cause of slow growth of the absorption coefficient with increasing the photon energy (see Fig. 1, region $a$ ).

The plate CdSe single crystals with the low dark resistivity $(\sim 1 \mathrm{Ohm} \cdot \mathrm{cm})$ were doped with donor type impurities. At the point of their location, interaction of electron with charged impurity increases. As a result, a hill of potential energy near bottom of the conduction band and a hollow near top of the valence band are formed, i.e. a covariant local change in potential energy takes place at the point of the donor impurity location (Fig. 3). As a result of limitation of charge carrier motion by the potential well walls, quantum-sized levels are formed in it. Their energy position and number depend on the point of localization and depth of the potential well. These levels are often referred to as fluctuation ones. This potential relief does not change with time, i.e. it is static. When the temperature of crystals is different from absolute zero, at any fixed time, in some points the crystal atoms approach to each other, and in other points they move away from each other to their equilibrium state. Because of the arbitrary value of displacement of lattice atoms caused by their thermal motion in the crystal, a chaotic set of power lattice disturbances and local electric fields is formed, i.e. the potential relief is created. A feature of this potential relief is its dynamic nature. That is, the depth and size of the potential wells at each given point of crystal is continuously changing in time.

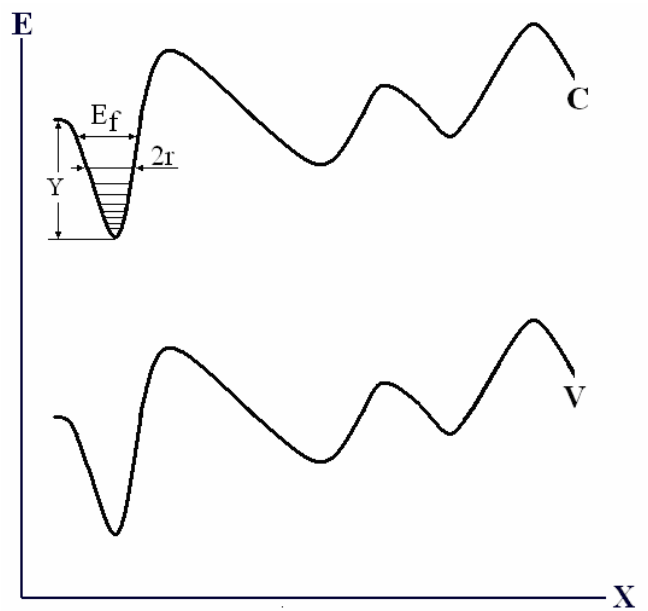

Fig. 3. Schematic diagram of the potential relief. $C$ is the bottom of the conduction band, $V$ is the top of the valence band, $Y-$ depth, and $r$ - radius of the potential well with the fluctuation levels, $E_{\mathrm{F}}$ is the energy position of the fluctuation level.
The presence of potential relief is manifested in creation of the density-of-state tail that adjoins to the conduction band in donor semiconductor (Fig. 4). This tail consists of two parts. One part is formed by perturbations of donor impurities, and the other part is caused by thermal vibrations of lattice atoms.

Absorption of light quanta in the density-of-state tail region is caused by transfer of electrons from the valence band to the fluctuation level in the tail. In this case (Fig. 1 region $b$ ), there is an exponential increase in the absorption coefficient, which is described by Urbach's rule when $h v<E_{g}[3-5]$ :

$K=K_{0} \exp \left[\frac{-\sigma\left(E_{g}-h v\right)}{k T_{0}}\right]$,

where $K_{0}$ and $\sigma$ are constants, $k$ is the Boltzmann constant, $E_{g}$ is the bandgap, $T_{0}$ is the absolute temperature.

Finally, when the photon energy $h v$ is spent on transferring electrons from the valence band to the energy levels of conduction band (Fig. 4), the absorption spectrum of the direct-band semiconductors is described by the root law

$$
K=A\left(h v-E_{g}\right)^{1 / 2}
$$

where $A$ is the constant.

Note that for both light polarizations, there are three clearly defined areas of light absorption (Fig. 1). The unique difference is that at the change of polarization from $E \perp C$ to $E \| C$, the spectrum shifts towards the high-energy side (compare the energy position of the curves 1 and 3 in Fig. 1).

\section{Edge absorption spectrum in the state of absorption saturation}

When single crystals are pumped by laser irradiation, the transmittance dependence on the intensity $I_{0}$ varies. For example, in the section $a$ (Fig. 2a) for all the wavelengths, the transmission $T$ does not depend on $I_{0}$. The reason for this is the presence of clusters that absorb or scatter light quanta nonradiatively.

In the sections $b$ and $c$, there is a characteristic dependence of $T$ on $I_{0}$ (Figs $2 \mathrm{~b}$ and 2c, respectively). The only difference of the dependences $T$ versus $I_{0}$ obtained for different wavelengths is a different value of the interval between transmission at low (where $T$ does not depend on $I_{0}$ ) and high (absorption saturation region, where $T$ also does not depend on $I_{0}$ ) intensities. Using the known value $T$ and formula (1), we obtain the value of the absorption coefficient for semiconductor in the linear optics field and in the absorption saturation state. It allows us to determine the absorption spectrum at low(Fig. 1, points on the curves 1 and 3 ) and high-intensity laser irradiation (Fig. 1, points on the curves 2, 4).

The absorption spectra in the saturation state are characterized by the following features. 


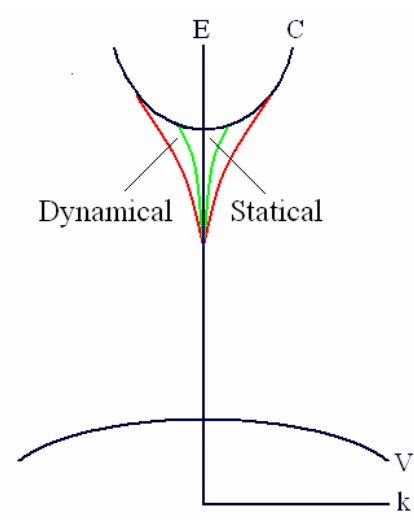

a

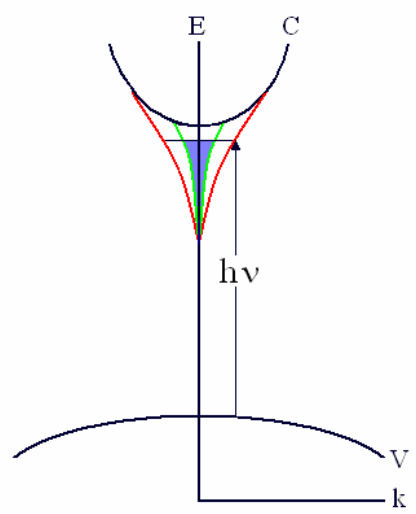

b

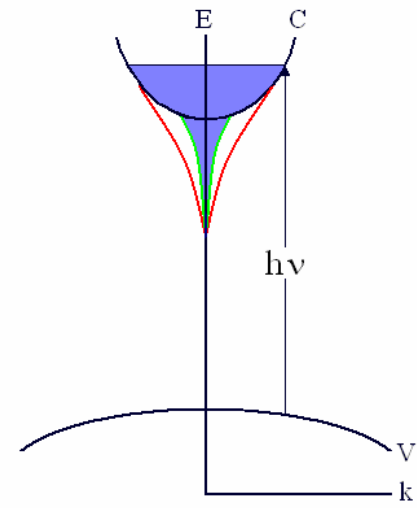

$\mathrm{c}$

Fig. 4. Simplified energy diagram. Here: $(a)$ without electron filling the density-of-state tails, $(b)$ filling the tail created by static disorder, (c) filling the conduction band and the tail created by static disorder. (In online version: green lines indicate the region of the tail created by static disorder, red lines - the region formed by dynamic disorder, blue colour indicates electron filled region.)

1. The region (formed by clusters) of the weak dependence of $K$ on $h v$ (section $a$ in Fig. 1) is slightly expanded. The reason for this is the lack of absorption caused by the electron filling the energy states of clusters and the presence of scattering the light quanta by large (as compared with the lattice parameter) inhomogeneities caused by the presence of cation or anion type clusters or inhomogeneities of the refractive index.

2. There is an exponential region (region $b$ in Fig. 1) that is shifted towards the high-energy side as compared to the absorption spectrum in linear optics field. The reason of it is as follows. As a result of light quanta absorption, electrons can reach the fluctuation level of static potential relief and consistently fill the levels of the density-of-state tail (Fig. 4b). If electrons reach the fluctuation levels of dynamic potential relief, due to its continuous change in time they are thrown out of the potential well to the conduction band and then fall to the fluctuation levels of static potential relief. That is, in the state of absorption saturation the density-of-state tail created by static disorder of potential relief is filled, and the density-of-state tail created by dynamic disorder of potential relief is not filled (Fig. 4b). It leads to an increase of slope of the dependence $\lg K=f(h v)$ in the Urbach region during transition from linear absorption to the state of absorption saturation (cf. curves 1 and 2 or 3 and 4).

3. When irradiated CdSe samples with photons, the energy of which is higher than the bandgap, electrons fill the tail created by static disorder and conduction band extremum (Fig. 4c). As a result, the absorption coefficient is reduced only to the level corresponding to light absorption in the tail, which is caused by dynamic disorder. This change indicates the weak dependence of $K$ on $h v$ in the transition region of the state of absorption saturation (Fig. 1, region $W$ ).
The process of filling the conduction band occurs as long as you begin the stimulated emission [8].

\section{Conclusion}

In the state of absorption saturation, the absorption edge shape is significantly different from the edge shape characteristic of the irradiation level in linear optics. In the state of absorption saturation, the edge shape is determined by the process of electron filling the densityof-state tails created by static disorder and the conduction band extremum as well as the lack of electron filling of the density-of-state tail formed by dynamic disorder.

\section{References}

1. Physics and Chemistry of $A^{I I} B^{V I}$ Compounds. Ed. by prof. S.A. Medvedev. Mir, Moscow, vol. 1, p. 1-624 (in Russian).

2. M. Schaffner, X. Bao, A. Penzkofer, Principal optical constants measurement of uniaxial crystal CdSe in the wavelength region between 380 and $950 \mathrm{~nm} / /$ Appl. Opt. 31(22), p. 4546-4552 (1982).

3. Y. Toyozawa, The Urbach rule and exciton-lattice interaction // Technical Reports. ISSP A, 1(119), p. 1-68 (1964).

4. D. Redfield, Electric fields of defects in solids // Phys. Rev. 130(3), p. 914-915 (1963).

5. J.D. Dow, D. Redfield, Urbach rule // Phys. Rev. $B$, 5(2), p. 594-599 (1972).

6. N.R. Kulish, A.F. Maznichenko, B.M. Bulakh, Optical absorption saturation in CdSe // Fizika i tekhnika poluprovodnikov, 12(5), p. 987-990 (1978), in Russian.

7. N.R. Kulish, N.I. Malysh, B.M. Bulakh, Dynamic effect by Burstein-Moss in CdSe // Ukrainskii fizicheskii zhurnal, 35(5), p. 671-674 (1990), in Russian.

8. A.W. Mol, R.A. Muribeca, E.A. Meneses, Stimulated photoluminescence of CdSe // Solid State Communs. 60(5), p. 423-425 (1986). 